\title{
A New Hilbert-type Inequality with the Homogeneous Kernel of Degree -2 and with the Integral
}

\author{
ZENG Zheng ${ }^{1}$, Prof. Dr. K. Raja Rama Gandhi ${ }^{2}$ and XIE Zitian ${ }^{3}$ \\ ${ }^{1}$ Shaoguan University, Shaoguan, Guangdong, 512005 P. R. China \\ ${ }^{2}$ Resource person in Math for Oxford University Press and Professor in Math at BITS-Vizag. \\ ${ }^{3}$ Zhaoqing University, Zhaoqing, Guangdong, 526061, P. R. China
}

Keywords: single parameter; weight function; Holder's inequality; equivalent form

Abstract. By using the weight functions and by means of Hadamard's inequality, we present a new Hilbert-type inequality with the integral in whole plane, a best constant factor and a homogeneous kernel of degree-2 .

\section{Introduction}

If $f(x), g(x) \geq 0$, such that $0<\int_{0}^{\infty} f^{2}(x) \mathrm{d} x<\infty$ and $0<\int_{0}^{\infty} g^{2}(x) \mathrm{d} x<\infty$, then

$$
\int_{0}^{\infty} \int_{0}^{\infty} \frac{f(x) g(y)}{x+y} \mathrm{~d} x \mathrm{~d} y<\pi\left\{\int_{0}^{\infty} f(x) d x \int_{0}^{\infty} g(x) \mathrm{d} x\right\}^{1 / 2}
$$

where the constant factor $\pi$ is the best possible. Inequality (1.1) is well-known as Hilbert's integral inequality, which has been extended by Hardy-Riesz as [2]

if $p>1,1 / p+1 / q=1, f(x), g(x) \geq 0$, such that $0<\int_{0}^{\infty} f^{p}(x) \mathrm{d} x<\infty$ and $0<\int_{0}^{\infty} g^{q}(x) \mathrm{d} x<\infty$ then we have the following Hardy-Hilbert's integral inequality:

$$
\int_{0}^{\infty} \int_{0}^{\infty} \frac{f(x) g(y)}{x+y} \mathrm{~d} x \mathrm{~d} y<\frac{\pi}{\sin (\pi / p)}\left(\int_{0}^{\infty} f^{p}(x) \mathrm{d} x\right)^{1 / p}\left(\int_{0}^{\infty} g^{q}(x) \mathrm{d} x\right)^{1 / q} .
$$

with the same best constant factor $\frac{\pi}{\sin (\pi / p)}$.

In recent years, inequalities (1.1) and (1.2) have many generalizations and variants. (1.1) has been strengthened by Yang and others. (including double series inequalities)[3-14].

In this paper, by using the way of weight function, we give a new Hilbert-type inequality with the integral in whole plane, a best constant factor and a homogeneous kernel of degree-2. We also consider its equivalent forms and the reverses.

In the following, we always suppose that: $1 / p+1 / q=1, p \neq 0, \theta \in(0, \pi)$.

\section{Some lemmas}

Lemma 2.1 Define the weight functions as follow:

$$
w(x):=\int_{-\infty}^{\infty} \frac{|x| d y}{(|x|+|y|) \sqrt{y^{2}+2 x y \cos \theta+x^{2}}}, \quad w(y):=\int_{-\infty}^{\infty} \frac{|y| d x}{(|x|+|y|) \sqrt{y^{2}+2 x y \cos \theta+x^{2}}} .
$$

Then, we have

$$
w(x)=w(y)=K:=\frac{1}{2 \sin \frac{\theta}{2}} \ln \frac{1+\sin \frac{\theta}{2}}{1-\sin \frac{\theta}{2}}+\frac{1}{2 \cos \frac{\theta}{2}} \ln \frac{1+\cos \frac{\theta}{2}}{1-\cos \frac{\theta}{2}}
$$


Proof. We only prove that $w(x)=K$ for $x \in(-\infty, 0)$.

Setting $y=t x$, and $t=(1-u) / u$ then[3]

$$
\begin{aligned}
& w(x)=\int_{-\infty}^{0} \frac{|x| d y}{(|x|+|y|) \sqrt{y^{2}+2 x y \cos \theta+x^{2}}}+\int_{0}^{\infty} \frac{(-x) d y}{(|x|+|y|) \sqrt{y^{2}+2 x y \cos \theta+x^{2}}}:=w_{1}+w_{2}, \\
& w_{1}=\int_{-\infty}^{0} \frac{d t}{(-x-y) \sqrt{y^{2}+2 x y \cos \theta+x^{2}}}=\int_{0}^{\infty} \frac{d u}{(1+t) \sqrt{t^{2}+2 t \cos \theta+1}} \\
& =\int_{0}^{1} \frac{\sqrt{(2-2 \cos \theta) u^{2}-(2-2 \cos \theta) u+1}}{\sqrt{1}} \\
& =\frac{1}{2 \sin \frac{\theta}{2}} \ln \left|\sqrt{(2-2 \cos \theta) u^{2}-(2-2 \cos \theta) u+1}+\sqrt{2-2 \cos \theta} u-\frac{2-2 \cos \theta}{2 \sqrt{2-2 \cos \theta}}\right|_{0}^{1} \\
& =\frac{1}{2 \sin \frac{\theta}{2}} \ln \frac{2 \sin \frac{\theta}{2}+1-\cos \theta}{2 \sin \frac{\theta}{2}+\cos \theta-1} \\
& =\frac{1}{2 \sin \frac{\theta}{2}} \ln \frac{1+\sin \frac{\theta}{2}}{1-\sin \frac{\theta}{2}}
\end{aligned}
$$

Setting $y=-t x$, then

$w_{2}=\int_{0}^{\infty} \frac{(-x) d y}{(-x+y) \sqrt{y^{2}+2 x y \cos \theta+x^{2}}}=\int_{0}^{\infty} \frac{d t}{(1+t) \sqrt{t^{2}-2 t \cos \theta+1}}$

$=\int_{0}^{\infty} \frac{d t}{(1+t) \sqrt{t^{2}+2 t \cos (\pi-\theta)+1}}$

$$
=\frac{1}{2 \cos \frac{\theta}{2}} \ln \frac{1+\cos \frac{\theta}{2}}{1-\cos \frac{\theta}{2}},
$$

and then

$$
w(x)=w_{1}+w_{2}=\frac{1}{2 \sin \frac{\theta}{2}} \ln \frac{1+\sin \frac{\theta}{2}}{1-\sin \frac{\theta}{2}}+\frac{1}{2 \cos \frac{\theta}{2}} \ln \frac{1+\cos \frac{\theta}{2}}{1-\cos \frac{\theta}{2}}=K .
$$

Easily if $x \in(0, \infty)$, then we have $w(x)=K$ and

$$
w(y)=K .
$$

The lemma is proved. 
Lemma 2.2 For $\frac{q}{4}>\varepsilon>0, q>1$ define both functions, $f$ and $g$ as follow:

$f(x)=\left\{\begin{array}{ll}x^{-2 \varepsilon / p}, & \text { if } x \in(1, \infty), \\ 0, & \text { if } x \in[-1,1], \\ (-x)^{-2 \varepsilon / p}, & \text { if } x \in(-\infty, 1),\end{array} \quad\right.$ and $g(x)= \begin{cases}x^{-2 \varepsilon / q}, & \text { if } x \in(1, \infty), \\ 0, & \text { if } x \in[-1,1], \\ (-x)^{-2 \varepsilon / q}, & \text { if } x \in(-\infty, 1) .\end{cases}$

Then

$$
\begin{gathered}
I(\varepsilon):=\varepsilon\left\{\int_{-\infty}^{\infty}|x|^{-1} f^{p}(x) d x\right\}^{1 / p}\left\{\int_{-\infty}^{\infty}|x|^{-1} g^{q}(x) d x\right\}^{1 / q}=1 ; \\
\tilde{I}(\varepsilon):=2 \varepsilon \int_{-\infty}^{\infty} \int_{-\infty}^{\infty} \frac{f(x) g(y)}{(|x|+|y|) \sqrt{y^{2}+2 x y \cos \theta+x^{2}}} d x d y=K+o(1)\left(\text { for } \varepsilon \rightarrow 0^{+}\right),
\end{gathered}
$$

Proof Easily, we have

$$
I(\varepsilon):=2 \varepsilon\left\{\int_{1}^{\infty} x^{-1} x^{-2 \varepsilon} d x\right\}^{1 / p}\left\{\int_{1}^{\infty} x^{-1} x^{-2 \varepsilon} d x\right\}^{1 / q}=1
$$

Let $y=-Y$, using $f(-x)=f(x), g(-x)=g(x)$, and

$$
\begin{aligned}
& f(-x) \int_{-\infty}^{\infty} \frac{g(y) d y}{(|x|+|y|) \sqrt{y^{2}-2 x y \cos \theta+x^{2}}}=f(x) \int_{-\infty}^{\infty} g(Y) \frac{g(Y) d Y}{(|x|+|Y|) \sqrt{Y^{2}+2 x Y \cos \theta+x^{2}}} \\
& \text { we find that }
\end{aligned}
$$

$$
\begin{gathered}
\tilde{I}(\varepsilon)=2 \varepsilon \int_{0}^{\infty} f(x)\left(\int_{-\infty}^{\infty} \frac{g(y) d y}{(|x|+|y|) \sqrt{y^{2}+2 x y \cos \theta+x^{2}}}\right) d x:=I_{1}+I_{2}, \\
I_{1}=2 \varepsilon \int_{1}^{\infty} x^{-2 \varepsilon / p}\left(\int_{-\infty}^{-1} \frac{(-y)^{-\frac{2 \varepsilon}{q}} d y}{(x-y) \sqrt{y^{2}+2 x y \cos \theta+x^{2}}}\right) d x, \\
I_{2}=2 \varepsilon \int_{1}^{\infty} x^{-2 \varepsilon / p}\left(\int_{1}^{\infty} \frac{y^{-\frac{2 \varepsilon}{q}} d y}{(x+y) \sqrt{y^{2}+2 x y \cos \theta+x^{2}}}\right) d x .
\end{gathered}
$$

Setting $y=x u$, then

$$
\begin{aligned}
& I_{1}=2 \varepsilon \int_{1}^{\infty} x^{-2 \varepsilon / p}\left(\int_{1}^{\infty} \frac{y^{-\frac{2 \varepsilon}{q}}}{(x+y) \sqrt{y^{2}-2 x y \cos \theta+x^{2}}} d y\right) d x \\
& =2 \varepsilon \int_{1}^{\infty} x^{-1-2 \varepsilon}\left(\int_{\frac{1}{x}}^{\infty} \frac{u^{-\frac{2 \varepsilon}{q}}}{(u+1) \sqrt{u^{2}-2 u \cos \theta+1}} d u\right) d x
\end{aligned}
$$




$$
\begin{aligned}
& =2 \varepsilon\left[\int_{1}^{\infty} x^{-1-2 \varepsilon}\left(\int_{1}^{\infty} \frac{u^{-\frac{2 \varepsilon}{q}}}{(u+1) \sqrt{u^{2}-2 u \cos \theta+1}} d u\right) d x\right. \\
& \left.+\int_{1}^{\infty} x^{-1-2 \varepsilon}\left(\int_{\frac{1}{x}}^{1} \frac{u^{-\frac{2 \varepsilon}{q}}}{(u+1) \sqrt{u^{2}-2 u \cos \theta+1}} d u\right) d x\right] \\
& =\int_{1}^{\infty} \frac{u^{-\frac{2 \varepsilon}{q}}}{(u+1) \sqrt{u^{2}-2 u \cos \theta+1}} d u+2 \varepsilon \int_{0}^{1} \frac{u^{-\frac{2 \varepsilon}{q}}}{(u+1) \sqrt{u^{2}-2 u \cos \theta+1}}\left(\int_{1 / u}^{\infty} x^{-1-2 \varepsilon} d x\right) d u \\
& =\int_{1}^{\infty} \frac{u^{-\frac{2 \varepsilon}{q}}}{(u+1) \sqrt{u^{2}-2 u \cos \theta+1}} d u+\int_{0}^{1} \frac{u^{\frac{2 \varepsilon}{p}}}{(u+1) \sqrt{u^{2}-2 u \cos \theta+1}} d u \\
& =\int_{0}^{\infty} \frac{d u}{(u+1) \sqrt{u^{2}-2 u \cos \theta+1}} \\
& +\int_{1}^{\infty} \frac{u^{-\frac{2 \varepsilon}{q}}-1}{(u+1) \sqrt{u^{2}-2 u \cos \theta+1}} d u+\int_{0}^{1} \frac{u^{\frac{2 \varepsilon}{p}}-1}{(u+1) \sqrt{u^{2}-2 u \cos \theta+1}} d u \\
& =\frac{1}{2 \cos \frac{\theta}{2}} \ln \frac{1+\cos \frac{\theta}{2}}{1-\cos \frac{\theta}{2}}+o(1)\left(\text { for } \varepsilon \rightarrow 0^{+}\right) .
\end{aligned}
$$

If fact we find

$\left|\frac{1-u^{-\frac{2 \varepsilon}{q}}}{(u+1) \sqrt{u^{2}-2 u \cos \theta+1}}\right| \leq \frac{1}{(u+1) \sqrt{u^{2}-2 u \cos \theta+1}}, u \in(1, \infty)$,

$$
\begin{gathered}
\int_{1}^{\infty} \frac{1}{(u+1) \sqrt{u^{2}-2 u \cos \theta+1}} d u \leq w_{2}<\infty, \\
\left|\frac{u^{\frac{2 \varepsilon}{p}}-1}{(u+1) \sqrt{u^{2}-2 u \cos \theta+1}}\right| \leq \frac{1}{(u+1) \sqrt{u^{2}-2 u \cos \theta+1}}, u \in(0,1), \\
\int_{0}^{1} \frac{1}{(u+1) \sqrt{u^{2}-2 u \cos \theta+1}} d u \leq w_{2}<\infty
\end{gathered}
$$

And by Lebesgue convergence theorem, we have the last expression. 
then we have the conclusion as follows:

$$
I_{1} \rightarrow w_{2}\left(\varepsilon \rightarrow 0^{+}\right),
$$

Similarly $I_{2} \rightarrow w_{1}$ (for $\varepsilon \rightarrow 0^{+}$), and we have

$$
\tilde{I}(\varepsilon)=I_{1}+I_{2}=K+o(1)\left(\text { for } \varepsilon \rightarrow 0^{+}\right) \text {. }
$$

The lemma is proved.

Lemma 2.3. If $p>1, f(x) \geq 0,0<\int_{-\infty}^{\infty}|x|^{-1} f^{p}(x) \mathrm{d} x<\infty$, then we have

$$
J:=\int_{-\infty}^{\infty}|y|^{p-1}\left(\int_{-\infty}^{\infty} \frac{f(x)}{(|x|+|y|) \sqrt{y^{2}+2 x y \cos \theta+x^{2}}} d x\right)^{p} d y \leq K^{p} \int_{-\infty}^{\infty}|x|^{-1} f^{p}(x) \mathrm{d} x .
$$

Proof By lemma 2.2,we find

$$
\begin{aligned}
& \left(\int_{-\infty}^{\infty} f(x)\left(\frac{1}{|x|+|y|) \sqrt{y^{2}+2 x y \cos \theta+x^{2}}}\right)^{\frac{1}{p}}\left(\frac{1}{(|x|+|y|) \sqrt{y^{2}+2 x y \cos \theta+x^{2}}}\right)^{\frac{1}{q}} d x\right)^{p} \\
\leq & \int_{-\infty}^{\infty} \frac{1}{|x|+|y|) \sqrt{y^{2}+2 x y \cos \theta+x^{2}}} f^{p}(x) d x\left(\int_{-\infty}^{\infty} \frac{1}{(|x|+|y|) \sqrt{y^{2}+2 x y \cos \theta+x^{2}}} d x\right)^{p-1} \\
= & K^{p-1}|y|^{-p+1} \int_{-\infty}^{\infty} \frac{f^{p}(x)}{(|x|+|y|) \sqrt{y^{2}+2 x y \cos \theta+x^{2}}} d x \\
J & K^{p-1} \int_{-\infty}^{\infty}\left[\int_{-\infty}^{\infty} \frac{f^{p}(x)}{(|x|+|y|) \sqrt{y^{2}+2 x y \cos \theta+x^{2}}} d x\right] d y \\
& =K^{p-1} \int_{-\infty}^{\infty}\left[\int_{-\infty}^{\infty} \frac{d y}{(|x|+|y|) \sqrt{y^{2}+2 x y \cos \theta+x^{2}}}\right] f^{p}(x) d x \\
= & K^{p} \int_{-\infty}^{\infty}|x|^{-1} f^{p}(x) \mathrm{d} x
\end{aligned}
$$

The lemma is proved.

\section{Main results}

Theorem 3.1 If both functions $f(x)$ and $g(x)$ are nonnegative measurable functions, and satisfy $0<\int_{-\infty}^{\infty}|x|^{-1} f^{p}(x) \mathrm{d} x<\infty$, and $0<\int_{-\infty}^{\infty}|x|^{-1} g^{q}(x) \mathrm{d} x<\infty$, then

$$
\begin{aligned}
I^{*}:= & \int_{-\infty}^{\infty} \int_{-\infty}^{\infty} \frac{f(x) g(y)}{(|x|+|y|) \sqrt{y^{2}+2 x y \cos \theta+x^{2}}} d x d y \\
& <K\left(\int_{-\infty}^{\infty}|x|^{-1} f^{p}(x) \mathrm{d} x\right)^{1 / p}\left(\int_{-\infty}^{\infty}|x|^{-1} g^{q}(x) \mathrm{d} x\right)^{1 / q},
\end{aligned}
$$


And

$$
J=\int_{-\infty}^{\infty}|y|^{p-1}\left(\int_{-\infty}^{\infty} \frac{f(x)}{(|x|+|y|) \sqrt{y^{2}+2 x y \cos \theta+x^{2}}} d x\right)^{p} d y<K^{p} \int_{-\infty}^{\infty}|x|^{-1} f^{p}(x) \mathrm{d} x
$$

Inequalities (3.1)and (3.2) are equivalent, and the constant factors in the two forms are all the best possible.

Proof If (2.5) takes the form of equality for some $y \in(-\infty, 0) \cup(0, \infty)$, then there exist constants $\mathrm{M}$ and $\mathrm{N}$, such that they are not all zero, and

$$
M f^{p}(x)=N \text { a.e. in }(-\infty, \infty) \text {. }
$$

We claim that $M \neq 0$, otherwise $N=0$, then $|x|^{-1} f^{p}(x)=N /(M|x|)$ a.e. in $(-\infty, \infty)$ which contradicts the fact that $0<\int_{-\infty}^{\infty}|x|^{-1} f^{p}(x) \mathrm{d} x<\infty$. Hence by (2.4), we have (3.2).

By Holder's inequality with weight and (3.2), we have,

$$
\begin{aligned}
& I^{*}=\int_{-\infty}^{\infty}\left[|y|^{1 / q} \int_{-\infty}^{\infty} \frac{f(x)}{(|x|+|y|) \sqrt{y^{2}+2 x y \cos \theta+x^{2}}} d x\right]\left[|y|^{-1 / q} g(y)\right] d y \\
& \leq(J)^{1 / p}\left(\int_{-\infty}^{\infty}|y|^{-1} g^{q}(y) \mathrm{d} y\right)^{1 / q} .
\end{aligned}
$$

Using (3.2), we have (3.1).

Setting

$$
g(y)=|y|^{p-1}\left(\int_{-\infty}^{\infty} \frac{f(x)}{(|x|+|y|) \sqrt{y^{2}+2 x y \cos \theta+x^{2}}} d x\right)^{p-1},
$$

$$
\text { , then } J=\int_{-\infty}^{\infty}|y|^{-1} g^{q}(y) \mathrm{d} y,
$$

by (2.5) we have $J<\infty$. If $J=0$ then (3.2) is proved; If $0<J<\infty$, Then by (3.1),we obtain

$$
\begin{gathered}
0<\int_{-\infty}^{\infty}|y|^{-1} g^{q}(y) \mathrm{d} y=J=I^{*}<K\left(\int_{-\infty}^{\infty}|x|^{-1} f^{p}(x) \mathrm{d} x\right)^{1 / p}\left(\int_{-\infty}^{\infty}|x|^{-1} g^{q}(x) \mathrm{d} x\right)^{1 / q}, \\
\left(\int_{-\infty}^{\infty}|x|^{-1} g^{q}(x) \mathrm{d} x\right)^{1 / p}=J^{1 / p}<K\left(\int_{-\infty}^{\infty}|x|^{-1} f^{p}(x) \mathrm{d} x\right)^{1 / p},
\end{gathered}
$$

namely (3.1)and (3.2) are equivalent.

If the constant factor $K$ in (3.1) is not the best possible, then there exists a positive number $h$ (with $h<K$ ), such that

$$
\int_{-\infty}^{\infty} \int_{-\infty}^{\infty} \frac{f(x) g(y)}{(|x|+|y|) \sqrt{y^{2}+2 x y \cos \theta+x^{2}}} d x d y<h\left(\int_{-\infty}^{\infty}|x|^{-1} f^{p}(x) \mathrm{d} x\right)^{1 / p}\left(\int_{-\infty}^{\infty}|x|^{-1} g^{q}(x) \mathrm{d} x\right)^{1 / q} .
$$

For $\varepsilon>0$,by (3.4), using lemma 2.2,we have

$$
\tilde{I}(\varepsilon)=k+o(1)<\varepsilon h\left(\int_{-\infty}^{\infty}|x|^{-1} f^{p}(x) \mathrm{d} x\right)^{1 / p}\left(\int_{-\infty}^{\infty}|x|^{-1} g^{q}(x) \mathrm{d} x\right)^{1 / q}=h .
$$

Hence we find $K+o(1)<h$. For $\varepsilon \rightarrow 0^{+}$it follows that $K \leq h$, which contradicts the fact that $h<K$. Hence the constant $h$ in (3.1) is the best possible. Since (3.1) and (3.2) are equivalent, if the constant factor in (3.2) is not the best possible, then by using (3.3), we can get a contradiction that the constant factor in (3.1) is not the best possible.

Thus we complete the proof of the theorem. 
Theorem 3.2 If $1>\mathrm{p}>0$, both functions $f(x)$ and $g(x)$ are nonnegative measurable functions, satisfying

$$
\begin{gathered}
0<\int_{-\infty}^{\infty}|x|^{-1} f^{p}(x) \mathrm{d} x<\infty \text { and } 0<\int_{-\infty}^{\infty}|x|^{-1} g^{q}(x) \mathrm{d} x<\infty \text {, then } \\
I^{*}:=\int_{-\infty}^{\infty} \int_{-\infty}^{\infty} \frac{f(x) g(y)}{(|x|+|y|) \sqrt{y^{2}+2 x y \cos \theta+x^{2}}} d x d y \\
\quad>K\left(\int_{-\infty}^{\infty}|x|^{-1} f^{p}(x) \mathrm{d} x\right)^{1 / p}\left(\int_{-\infty}^{\infty}|x|^{-1} g^{q}(x) \mathrm{d} x\right)^{1 / q} \\
J=\int_{-\infty}^{\infty}|y|^{p-1}\left(\int_{-\infty}^{\infty} \frac{f(x)}{(|x|+|y|) \sqrt{y^{2}+2 x y \cos \theta+x^{2}}} d y>K^{p} \int_{-\infty}^{\infty}|x|^{-1} f^{p}(x) \mathrm{d} x\right.
\end{gathered}
$$

and

$$
L:=\int_{-\infty}^{\infty}|x|^{q-1}\left(\int_{-\infty}^{\infty} \frac{f(x)}{(|x|+|y|) \sqrt{y^{2}+2 x y \cos \theta+x^{2}}} g(y) d y\right)^{q} d x<K^{q} \int_{-\infty}^{\infty}|y|^{-1} g^{q}(y) d y .
$$

Inequalities (3.5),(3.6)and (3.7) are equivalent, and the constant factors $K, K^{p}$ and $K^{q}$ are the best possible.

Proof By the reverse Holder's inequality and the same way, we can obtain the reverse forms of (2.5)and (3.3). And then we deduce (3.6), by the some way, we obtain (3.5).

Setting $\mathrm{g}(\mathrm{y})$ as Theorem3.1, we obtain $\mathrm{J}>0$. If $J=\infty$, then we have (3.6) if $0<J<\infty$, then by (3.5)

$$
\int_{-\infty}^{\infty}|y|^{-1} g^{q}(y) d y=J=I^{*}>K\left(\int_{-\infty}^{\infty}|x|^{-1} f^{p}(x) d x\right)^{1 / p}\left(\int_{-\infty}^{\infty}|x|^{-1} g^{q}(x) d x\right)^{1 / q} .
$$

$\underset{\text { Detting }}{J^{\frac{1}{q}}}$ in the above, and we have (3.6), Hence inequalities
\[ f(x)=|x|^{q-1} \int_{-\infty}^{\infty} \frac{g(y)}{(|x|+|y|) \sqrt{y^{2}+2 x y \cos \theta+x^{2}}} d y \]

By the same way, we find

$$
\begin{gathered}
\infty>\int_{-\infty}^{\infty}|x|^{-1} f^{p}(x) \mathrm{d} x=L=\int_{-\infty}^{\infty} \int_{-\infty}^{\infty} \frac{f(x) g(y)}{(|x|+|y|) \sqrt{y^{2}+2 x y \cos \theta+x^{2}}} d x d y \\
>K\left(\int_{-\infty}^{\infty}|x|^{-1} f^{p}(x) \mathrm{d} x\right)^{1 / p}\left(\int_{-\infty}^{\infty}|x|^{-1} g^{q}(x) \mathrm{d} x\right)^{1 / q} \\
\int_{-\infty}^{\infty}|x|^{-1} f^{p}(x) \mathrm{d} x=L<K^{q} \int_{-\infty}^{\infty}|x|^{-1} g^{q}(x) \mathrm{d} x,
\end{gathered}
$$

and we have (3.7). By the reverse Holder's inequality, we have

$$
\begin{aligned}
I^{*}=\int_{-\infty}^{\infty}\left[|x|^{-\frac{1}{p}} f(x)\right]\left[\int_{-\infty}^{\infty}|x|^{\frac{1}{p}} g(y) \frac{f(x)}{(|x|+|y|) \sqrt{y^{2}+2 x y \cos \theta+x^{2}}} d y\right] d x \\
\geq\left(\int_{-\infty}^{\infty}|x|^{-1} f^{p}(x) d x\right)^{1 / p} L^{1 / q} .
\end{aligned}
$$


Then by (3.7), we have (3.5), which is equivalent to (3.7).Therefore (3.5)-(3.7)are equivalent.

If there exist a constant $h^{*}>K$, such that (3.5) still valid as we replace $K$ by $h^{*}$, the by the reverse of (3.4) we have: $K \geq h^{*}\left(\varepsilon \rightarrow 0^{+}\right)$,

Hence $h^{*}=K$ is the best value of (3.5). We conform that the constant factor of (3.6)(3.7) is the best possible, otherwise by the reverse of (3.3)(3.10), we can get a contradiction that the constant factor in (3.5)is not the best possible.

Remark. For $\theta=\frac{\pi}{4}$ in (3.1),we have

$$
\begin{gathered}
\int_{-\infty}^{\infty} \int_{-\infty}^{\infty} \frac{f(x) g(y)}{(|x|+|y|) \sqrt{y^{2}+\sqrt{2} x y+x^{2}}} d x d y \\
<2 \sqrt{2} \ln (\sqrt{2}+1)\left(\int_{-\infty}^{\infty}|x|^{-1} f^{p}(x) \mathrm{d} x\right)^{1 / p}\left(\int_{-\infty}^{\infty}|x|^{-1} g^{q}(x) \mathrm{d} x\right)^{1 / q}
\end{gathered}
$$

Foundation iterm: This work is supported by the Natural Science Funds of Guangdong Province, (No. S2012010010069)

\section{References}

[1] Hardy G.H.,Littlewood J E.and Polya G, Inequalities, Cambridge University Press, Cambridge, 1952.

[2] Hardy G. H., Note on a theorem of Hilbert concerning series of positive terems, Proceedings London Math. Soc, 1925,23(2): Records of Proc. XLV-XLVI.

[3] Zitian Xie, A Hilbert-type integral inequality with the kernel of irrational expression, Mathematics in practice and theory, 2008,38 (16) : 128-133

[4] Zitian Xie and Zeng Zheng,A Hilbert-type integral inequality whose kernel Is a homogeneous form of degree -3.Math.Anal.Appl.,2008,339;324-331

[5] Bicheng Yang, A new Hilbert-type integral inequality with some parameters,Journal of Jilin University(Science Edition), 2008,46(6) : 1085-1090.

[6] Xie Zitian, A New Hilbert-type integral inequality with the homogeneous kernel of real numberdegre, Journal of Jishou University(Naturnal Science Edition), 2011,32(4), 26-30

[7] Xie Zitian,Zeng Zheng, A New Hilbert-Type integral inequality with the Homogeneous Kernel of Degree -2 and with the Integral in Whole Plane, Journal of Applied Mathematics and Bioinformatics,2012,2(1),29-39.

[8] Zheng Zeng and Zitian Xie, On a new Hilbert-type integral inequality with the the integral in whole plane,Journal of Inequalities and Applications , vol. 2010, Article ID 256796, 8 pages, 2010. doi:10.1155/2010/256796

[9] Zitian Xie,Bicheng Yang, Zheng Zeng,A New Hilbert-type integral inequality with the homogeneous kernel of real number-degree ,Journal of Jilin University(Science Edition), 2010,48(6)941-945.

[10] Zitian Xie and Benlu Fu, Xie Zitian,Zeng Zheng, On a Hilbert-type integral inequality with the homogeneous kernel of real number-degree and its operator form, Advances and Applications in Mathematical Sciences 2011,10(5),481-490 
[11] Xie Zitian,Zeng Zheng, A new half-discrete Hilbert-type inequality with the homogeneous kernel of degree $-4 \mu$, Journal of Jishou University(Naturnal Science Edition), 2012,33 (2) 15-19.

[12] Xie Zitian,Zeng Zheng, On a Hilbert-type integral inequality with the homogeneous kernel of real number-degree and its operator form, Advances and Applications in Mathematical Sciences 2011,10(5),481-490

[13] Zitian Xie,Zheng Zeng,Qinghua Zhou ,A new Hilbert-type integral inequality with the homogeneous kernel of real number-degree and its equivalent inequality forms,Journal of Jilin University(Science Edition), 2012,50(4), 693-697.

[14] Xie Zitian,K. Raja Rama Gandhi,Zeng Zheng,A new Hilbert-type integral inequality with the homogeneous kernel of real degree form and the integral in whole plane,Bulletin of Society for Mathematical Services \& Applications, Vo2. No.1,2013,95-109. 